\title{
The relationship between repetition suppression and face perception
}

Petra Hermann ${ }^{1,2}$, Mareike Grotheer ${ }^{3,4}$, Gyula Kovács ${ }^{3,4,5, *}$, Zoltán Vidnyánszky $^{1,5, *}$

${ }^{1}$ Brain Imaging Centre, Research Centre for Natural Sciences, Hungarian Academy of Sciences, Budapest, Hungary

${ }^{2}$ Faculty of Information Technology and Bionics, Pázmány Péter Catholic University, Budapest, Hungary

${ }^{3}$ Institute of Psychology, Friedrich-Schiller-University of Jena, Jena, Germany ${ }^{4}$ DFG Research Unit Person Perception, Friedrich-Schiller-University of Jena, Jena, Germany

${ }^{5}$ Department of Cognitive Science, Budapest University of Technology and Economics, Budapest, Hungary

*These authors contributed equally.

\section{Corresponding Author:}

Petra Hermann

Brain Imaging Centre

Research Centre for Natural Sciences

Hungarian Academy of Sciences

Magyar tudósok körútja 2.

1117 Budapest

Hungary

+36-1-382-6904

hermann.petra@ttk.mta.hu

Author contributions: P.H., G.K., Z.V. designed research; P.H., G.K., M.G., performed research; P.H., M.G., G.K., Z.V. analyzed data; P.H., M.G., G. K., and Z.V. wrote the paper. 


\begin{abstract}
Repetition of identical face stimuli leads to fMRI response attenuation (fMRI adaptation, fMRIa) in the core face-selective occipito-temporal visual cortical network, involving the bilateral fusiform face area (FFA) and the occipital face area (OFA). However, the functional relevance of fMRIa observed in these regions is unclear as of today. Therefore, here we aimed at investigating the relationship between fMRIa and face perception ability by measuring in the same human participants both the repetition-induced reduction of fMRI responses and identity discrimination performance outside the scanner for upright and inverted face stimuli. In the correlation analysis the behavioral and fMRI results for the inverted faces were used as covariates to control for the individual differences in overall object perception ability and basic visual feature adaptation processes, respectively. The results revealed a significant positive correlation between the participants' identity discrimination performance and the strength of fMRIa in the core face processing network, but not in the extrastriate body area (EBA). Furthermore, we found a strong correlation of the fMRIa between OFA and FFA and also between OFA and EBA, but not between FFA and EBA. These findings suggest that there is a face-selective component of repetition-induced reduction of fMRI responses within the core face processing network, which reflects functionally relevant adaptation processes involved in face identity perception.
\end{abstract}

Keywords: repetition suppression, fMRI adaptation, face discrimination, FFA, OFA

\title{
Introduction
}

When a sensory stimulus is repeated, the evoked neural signal is invariably smaller than the one observed for its first presentation. This phenomenon is observed for many sensory modalities and for various stimuli using different methods. For example, the response of the visually sensitive neurons of the inferior temporal cortex (IT) of the macaque brain decreases when a stimulus is repeated (Baylis and Rolls 1987; Kaliukhovich and Vogels 2011, 2012; Miller, Gochin, et al. 1991; Miller, Li, et al. 1991; Ringo 1996; Sawamura et al. 2006; Sobotka and Ringo 1994), an effect is termed repetition suppression (RS). Similarly, in functional magnetic resonance imaging (fMRI) experiments stimulus repetitions lead to the reduction of the blood oxygenation leveldependent (BOLD) signal when compared with non-repeating stimuli (Henson and Rugg 2003), a phenomenon called fMRI adaptation (fMRIa). Although a large number of studies tested the 
neural mechanisms of RS in the last decades, there are still several open questions regarding this phenomenon. While prior studies typically connected RS to local or entirely bottom-up mechanisms, such as fatigue, sharpening, or response facilitation (for a review see Grill-Spector et al. 2006), recent studies emphasized the role of top-down factors, such as predictions and expectations (Summerfield et al. 2008). Although current single-cell recording results suggest that a simple fatigue-related adaptation of the firing rate is, indeed, unable to explain RS related phenomena (Vogels 2016), the role of top-down effects is currently under heavy debate (for reviews see Auksztulewicz and Friston 2016; Grotheer and Kovács 2016; Kovács and Vogels 2014). The few available human neurochemical studies suggest the role of gamma-aminobutyricacid and acetylcholine in modulating neural responses during stimulus repetitions (for a review see Bunzeck and Thiel 2016).

The behavioral relevance of the neural RS remains an interesting and open question. RS is generally believed to reflect short-term plastic processes of the neurons, as they adapt to the temporal context of the current environment. Thereby RS reflects the flexibility of the neural system and its ability to adjust to continuously changing requirements, optimizing the performance of the individual (Henson 2003). However, so far we have only limited evidence of the direct relationship between RS and behavioral performance changes (Horner and Henson 2011). Previously, a long tradition of research connected RS to behavioral priming effects. Priming (Magnussen 2000) is a phenomenon when the prior presentation of a related or identical stimulus leads to faster and more accurate responses for the target. Indeed, recent neuroimaging studies found that trials leading to behavioral priming also lead to fMRIa in the fusiform and occipital face areas (FFA and OFA) of the human brain (Kaiser et al. 2013). Similarly, repetition priming has also been related to fMRIa in several cortical areas for objects and scenes, including prefrontal, parietal, and occipito-temporal ones (Soldan et al. 2010; Ward et al. 2013). However, a clear causal relationship could not been determined between behavioral priming and RS as of today. In fact, recent studies have raised doubts about the relationship of RS and priming: RS was either not specific to the "primed" conditions (Ganel et al. 2006) or the magnitude of RS did not correlate with the amount of behaviorally observed priming effects (Sayres and Grill-Spector 2006). These results suggest that RS and priming might co-occur but they are not necessarily connected to each other functionally. 
A more direct approach for investigating the behavioral relevance of RS would be to test whether the individual fMRIa effect is related to the face perception ability of the participants. GilaieDotan et al. (2010) found that the fMRI responses in the FFA to repeated face presentations varying in identity similarity were associated with the perceived face similarity. Indeed, in patients of acquired prosopagnosia (an inability to recognize faces) due to the lesion of OFA, a lack of RS has been found in the FFA (Schiltz et al. 2006; Steeves et al. 2009), suggesting its functional relevance. Furthermore, another study, measuring intracerebral EEG, found strong RS for face identity in the right OFA on an electrode, whose stimulation disrupted behavioral face discrimination (Jonas et al. 2014).

To test this relationship more directly, in the current study we investigated the association of fMRIa with the perceptual sensitivity of the participants for face stimuli in an identity discrimination task. We reasoned that if RS (and the consequent fMRIa) indeed reflects the better responding capacity of the neural system then this should manifest on the perceptual level as well. For this end, we compared the magnitude of fMRIa within areas of the core face network as well as the extrastriate body area (EBA, Downing et al. 2001) as a control area, with the behavioral performance of the participants in a face identity discrimination task.

\section{Materials and methods}

\section{Subjects}

Altogether 30 Caucasian subjects (8 male; 2 left-handed; mean age ( \pm SD): 22.8 (3.2) years) participated in the experiment and gave informed written consent in accordance with the protocols approved by the Ethical Committee of the Friedrich Schiller University Jena. One subject had to be excluded from the study due to technical difficulties in the data acquisition. Therefore, the present results are based on the data of 29 subjects. None of them had any history of neurological or psychiatric diseases, and all had normal or corrected-to-normal visual acuity.

\section{Behavioral experiment}

To determine the face perception ability of the participants, they performed a three-alternative forced choice perceptual face identity discrimination task outside the scanner (Fig. 1). 


\section{Stimuli}

In the psychophysical experiment, trials consisted of triplets of morphed faces. Front-view grayscale images of Caucasian male faces with neutral expression were cropped to eliminate external features and were equated for luminance and contrast. Triplets (Fig. 1) were obtained by first pairing two individuals and creating a linear morph continuum using a warping algorithm (JPsychoMorph, Tiddeman et al. 2001). Altogether 78 continua were created from thirteen individuals. Triplet members were selected from these continua as follows: face A and C were chosen to be the $20 / 80 \%$ and $80 / 20 \%$ points of the morph-space, respectively, while face B was taken from in between, such that the morph distance between the oddball image (e.g. C) and its neighbor (e.g. B) was larger than the distance between the other two images (e.g. A and B). This distance was based on pilot measurements to keep performance within the $60-70 \%$ range. Faces were presented upright and upside-down (inverted).

Triplets were displayed at the center of the screen on a uniform gray background in a regular triangular arrangement with $4^{\circ}$ of visual angle eccentricity. Triplets measured approximately $11^{\circ}$ $\times 12^{\circ}$, faces subtending $4.5^{\circ} \times 6.0^{\circ}$ each, and were presented on a CRT display at a refresh rate of $60 \mathrm{~Hz}$ viewed from $85 \mathrm{~cm}$. Stimulus presentation was controlled by MATLAB R2010a (The MathWorks Inc., Natick, MA, USA) using the Psychophysics Toolbox Version 3 (Brainard 1997; Pelli 1997) (http://psychtoolbox.org/).

\section{Experimental procedure}

Participants performed a three-alternative forced choice identity discrimination task. Subjects were required to select the face that differed the most from the other two (i.e. the oddball face). Each trial began with a cue $\left(1^{\circ}\right)$ appearing just above fixation for $100 \mathrm{~ms}$, indicating the orientation of the upcoming stimuli (upright or inverted). Triplets were presented without a fixation dot under free-viewing conditions until subjects responded; trials were terminated after $5000 \mathrm{~ms}$ if no response had been made. Trials were separated by an inter-trial interval, which varied randomly between 900 and $1100 \mathrm{~ms}$, with only the fixation dot present. Oddball faces appeared with equal probability in each of the three possible spatial positions. Each unique face triplet was presented only once per condition, randomly assigned to one of the three runs for each participant. Within a single run, the two conditions (upright/inverted) were intermixed and 
presented in random order. Each participant completed three runs, yielding 78 trials altogether for each condition.

\section{fMRI experiment}

The fMRI data analyzed in the current study was a subset of what we previously used to examine the face processing stages at which repetition probability affects fMRI adaptation (Grotheer et al. 2014). However, here we aimed at investigating the functional relevance of repetition suppression in face processing. Therefore, we only analyzed data from blocks with high stimulus repetition probabilities where the repetition suppression effect was clearly evident and the largest.

\section{Stimulation and Procedure}

The experimental design - as also described in Grotheer et al. (2014) - was similar to that of Summerfield et al. (2008) and to that of Kovács et al. (2012). Briefly, 240 grayscale, digital photos of full-frontal Caucasian faces, similar to the face stimuli of Kovács et al. (2012) and Kovács et al. (2013), were fit behind a circular mask (diameter $=5.5^{\circ}$ ) and either presented upright, or inverted in different runs of fMRI recordings. No stimulus occurred in more than one trial during each run. Stimuli were placed in the center of the screen on a uniform gray background. A trial contained two faces presented subsequently for $250 \mathrm{~ms}$ each, separated by an inter-stimulus interval that varied between 400 and $600 \mathrm{~ms}$ and was followed randomly by a 1 or $2 \mathrm{sec}$ long inter-trial interval. The first stimulus (S1), was either identical to (Repetition Trial, RepT) or different from the second stimulus (S2) (Alternation Trial, AltT). All stimuli subtended $2.75^{\circ}$ in non-target trials, target stimuli were reduced in size by $54 \%$. To reduce local feature adaptation the size of either S1 or S2 (chosen randomly) was reduced by $18 \%$. Stimuli were backprojected via an LCD video projector (NEC GT 1150, NEC Deutschland GmbH, Ismaning, Germany, with modified lens for short focal point) onto a translucent circular screen, placed inside the scanner bore. Presentation was controlled via MATLAB R2013a (The MathWorks Inc., Natick, MA, USA), using Psychophysics Toolbox Version 3 (Brainard 1997; Pelli 1997) (http://psychtoolbox.org/).

In addition to the different trial types, two different types of blocks were presented to the subjects with varying repetition probabilities (Grotheer et al. 2014). For the current study we only analyze 
the blocks where stimulus repetitions had high-probabilities (RepB). In these RepB 75\% of the non-target trials were RepT while $25 \%$ were AltT. As we did not observe reliable fMRIa within the alternating blocks (AltB), having inverse proportions of AltT and RepT (see Fig. 3b of Grotheer et al. 2014), these blocks were not analyzed in the current study. With the exception of the first four trials, which always consisted out of the more frequent trial type of that specific block (RepT in RepB and AltT in AltB), RepT and AltT were presented randomly within the blocks. In addition, $20 \%$ of all trials were target trials, whereas target trials could be AltT or RepT with the same relative probability. Hence, overall, Repetition Blocks consisted of $70 \%$ RepT and 30\% AltT (identical to the paradigm of Summerfield et al. (2008)). Both Alternation and Repetition Blocks contained 20 trials and were repeated 4 times during each run, so that a run contained 160 trials. The different blocks were separated from each other by a $7 \mathrm{sec}$ pause during which the phrase "Short Break" was presented centrally together with a countdown. The participants' task was to maintain central fixation throughout the trials and to signal the occurrence of target stimuli by pressing a button. Four runs were presented in total, whereas the order of upright and upside-down runs was counterbalanced across subjects.

\section{Imaging Parameters and Data Analysis}

A 3-Tesla MR scanner (Siemens Magnetom Trio, Erlangen, Germany) was used to perform the study. During the functional blocks we continuously acquired images (34 slices, $10^{\circ}$ tilted relative to axial, T2 $*$-weighted EPI sequence, TR $=2000 \mathrm{~ms}$; TE $=30 \mathrm{~ms}$; flip angle $=90^{\circ} ; 64 \mathrm{x}$ 64 matrices; in-plane resolution: $3 \times 3 \mathrm{~mm}$; slice thickness: $3 \mathrm{~mm} ; 20 \%$ inter-slice interval). To additionally obtain a 3D structural scan, high-resolution sagittal T1-weighted images were acquired using a 3D-MPRAGE sequence $(\mathrm{TR}=2300 \mathrm{~ms}$; $\mathrm{TE}=3.03 \mathrm{~ms} ; 1 \mathrm{~mm}$ isotropic voxel size).

The analysis of the imaging data differs considerably from the one performed in our previous report (Grotheer et al. 2014). Preprocessing and analysis of the imaging data were performed using the SPM12 toolbox (Wellcome Trust Centre for Neuroimaging) as well as custom-made scripts running on MATLAB 2013b (The MathWorks Inc., Natick, MA, USA). The functional images were spatially realigned to the first EPI image within a session for motion correction. The anatomical T1 image was coregistered to the mean functional T2*-weighted image generated during the realignment step followed by segmentation and normalization to the MNI-152 space 
using the new unified segmentation-normalization tool of SPM12. To spatially normalize functional images to MNI space we applied the deformation field parameters that were obtained during the normalization of the anatomical T1 image. After the normalization procedure, functional images were spatially smoothed with an 8-mm full-width at half-maximum isotropic Gaussian kernel. The gray matter mask derived from the segmentation of the anatomical image was used to restrict statistical analysis on the functional files. The 4 experimental conditions (AltB_AltT, AltB_RepT, RepB_AltT, RepB_RepT) as well as the target trials from our eventrelated sessions with upright and inverted faces were defined as separate regressors, which were convolved with the canonical Hemodynamic Response Function (HRF) of SPM12, for a General Linear Model (GLM) analysis of the data. Movement-related variance was accounted for by the spatial parameters resulting from the motion correction procedure. A high-pass filter with a cycle-cutoff of $128 \mathrm{~s}$ was also implemented in the design to remove low-frequency signals. The prepared regressors were then fitted to the observed functional time series within the cortical areas defined by the gray matter mask. In order to specifically address the questions that we aimed to investigate in the current study, we used parameter estimates (i.e. beta weights) only for the RepB_AltT and RepB_RepT conditions. To obtain beta weights for AltT and RepT in RepB with equal number of trials, we used a bootstrap procedure (resampling without replacement, $\mathrm{n}=$ 1000) where for RepT 4 non-target trials were randomly chosen from each of the 4 RepBlocks yielding 16 RepT per run which was treated as a separate regressor in the GLM approach. This procedure was repeated 1000 times and the resulting 1000 beta weights were averaged for each condition and subject for further analysis.

\section{ROI definition}

A separate functional localizer run (640 s long; $20 \mathrm{~s}$ epochs of faces, objects, human bodies and Fourier randomized versions of faces (Dakin et al. 2002), interleaved with $20 \mathrm{~s}$ of blank periods; $2 \mathrm{~Hz}$ stimulus repetition rate; $300 \mathrm{~ms}$ exposition time; $200 \mathrm{~ms}$ blank) was used to determine regions of interest (ROIs) for each participant. The fusiform face area (FFA) in the mid-fusiform gyrus was identified as an area responding more strongly to faces than objects and Fourier randomized versions of faces. The occipital face area (OFA) in the inferior occipital gyrus was determined as an area showing significantly stronger activation to faces than Fourier randomized versions of faces, while the extrastriate body area (EBA) localized in the lateral occipital cortex 
was determined as an area responding more intensely to human bodies relative to objects. Peak voxel activity of all regions of interest was required to meet a minimum threshold of $\mathrm{p}_{\text {uncorrected }}=$ 0.001. It was possible to define right FFA in 27, left FFA in 22 subjects (average MNI coordinates \pm SE: $40 \pm 0.7,-52 \pm 1.4,-18 \pm 0.6$ and $-39 \pm 0.5,-54 \pm 1.3,-20 \pm 0.6$, for right and left hemispheres, respectively), while right and left OFA was found in 24 subjects $(42 \pm 0.7,-77 \pm 1.1$, $-10 \pm 0.9$ and $-38 \pm 0.6,-80 \pm 1.1,-13 \pm 0.7)$. Right EBA could be defined in 26 and left EBA in 24 participants $(52 \pm 0.7,-70 \pm 0.9,7 \pm 0.8$ and $-49 \pm 0.8,-75 \pm 1.1,8 \pm 0.9)$.

Statistical analysis

To determine the magnitude of fMRIa in these regions, mean beta weights for AltT and RepT in the Repetition Blocks were extracted from a 6-mm-radius sphere around the peak voxel of each individually defined ROI, and entered into a two-way ANOVA with hemisphere (L vs. R) and trial (AltT vs. RepT) as within-subject factors. To examine whether fMRIa measured in the investigated ROIs is related to face-selective perceptual ability, we correlated the individual fMRIa magnitudes - calculated by subtracting beta weights of RepT from that of AltT - with subjects' performance on face identity discrimination. To control for the individual differences in low-level visual feature processing and overall object perception ability, we regressed out the fMRIa and identity discrimination performance for inverted faces from those for upright faces, respectively, before calculating correlations between these two measures. Subjects with behavioral face inversion effect $(\mathrm{N}=4$; calculated by subtracting performance for inverted faces from that for upright faces) below one standard deviation $(\mathrm{SD}=0.105)$ from the group mean (mean $=0.084)$ were excluded from this correlation analysis. These subjects had higher performance for inverted faces than upright faces which suggests poor face-selective processing or insufficient task engagement. Since the assumption of bivariate normality was fulfilled for all correlation pairs investigated ( $\mathrm{HZ}<0.20, \mathrm{p}>0.372$, Trujillo-Ortiz et al. 2007), Skipped Pearson's correlation coefficients were calculated with the Robust Correlation Toolbox (Pernet et al. 2012) in MATLAB. Bivariate outliers were detected using an adjusted box-plot rule and removed in the computation of skipped correlations. For correlation coefficients (r), 95\% confidence intervals (CI) were calculated based on 1000 samples with the percentile bootstrap method implemented in the toolbox. Correlation strengths were compared with the test proposed by Zou (2007). Using this method two-sided 95\% CIs were constructed for a difference between 
two dependent overlapping correlations based on their bootstrapped CI-s derived from the robust correlation analysis. Participants with missing data from any of the variables of interest were excluded for a given analysis.

\section{Results}

Behavioral results

Participants performed the three-alternative forced-choice identity-discrimination task with 61.5 $\pm 1.8 \%$ (mean percent correct \pm SEM) accuracy and showed a robust face inversion effect (FIE, Yin 1969), i.e. significantly higher performance for upright as compared to inverted faces $\left(\mathrm{t}_{(28)}=\right.$ $4.32, \mathrm{p}<0.001)$. When testing for the correlation between individual differences in face-selective perceptual ability and fMRIa, performance for inverted faces was used as a covariate to control for the individual differences in overall visual object perception.

\section{fMRI adaptation}

Fig. 2 presents the average BOLD signal separately for AltT and RepT in the case of upright (Fig. 2a) and inverted faces (Fig. 2b). In agreement with previous results (Andrews and Ewbank 2004; Ewbank et al. 2013; Kovács et al. 2012; Larsson and Smith 2012; Summerfield et al. 2008; Winston et al. 2004; Yovel and Kanwisher 2005), we found significant fMRIa, i.e. reduced BOLD signal for repeated (RepT) as compared to alternating faces (AltT) in the FFA and a moderate fMRIa in the OFA for upright faces (main effect of trial type for upright faces: $\mathrm{F}_{(1,21)}=$ 8.25, $\mathrm{p}=0.009$ and $\mathrm{F}_{(1,21)}=3.10, \mathrm{p}=0.093$ for the FFA and OFA, respectively) and also significant fMRIa in these regions for inverted faces (main effect of trial type for inverted faces: $\mathrm{F}_{(1,21)}=22.54, \mathrm{p}<0.001$ and $\mathrm{F}_{(1,21)}=9.89, \mathrm{p}=0.005$ for the FFA and OFA, respectively), as we reported in our previous work (Grotheer et al. 2014). In addition, fMRI responses in the right hemisphere were more pronounced compared to the left hemisphere for each condition in the case of both upright (main effect of hemisphere for upright faces: $F_{(1,21)}=15.81, p=0.001$ and $\mathrm{F}_{(1,21)}=13.35, \mathrm{p}=0.002$ for the FFA and OFA, respectively) and inverted faces (main effect of hemisphere for inverted faces: $\mathrm{F}_{(1,21)}=18.03, \mathrm{p}<0.001$ and $\mathrm{F}_{(1,21)}=5.35, \mathrm{p}=0.031$ for the FFA and OFA, respectively) indicating the right hemisphere dominance in face processing (Hecaen and Angelergues 1962; Kanwisher et al. 1997; Meadows 1974; Pitcher et al. 2007; Rossion et al. 
2012; Yovel et al. 2008). We also measured fMRIa in a region outside the typical face processing network, specifically in the extrastriate body area (EBA), which served as a control region. The EBA showed a significant BOLD response reduction in the case of RepT relative to AltT for upright (main effect of trial type for upright faces: $\mathrm{F}_{(1,23)}=4.56, \mathrm{p}=0.044$ ) and inverted faces (main effect of trial type for inverted faces: $\mathrm{F}_{(1,23)}=5.61, \mathrm{p}=0.030$ ) as well. The interaction of trial-type and hemisphere was not significant for any of the tested areas in the case of both upright ( $\mathrm{F}<1.08, \mathrm{p}>0.310$ for all comparisons) and inverted faces $(\mathrm{F}<1.88, \mathrm{p}>0.190$ for all comparisons). When testing for the correlation between individual differences in the magnitude of face-specific fMRIa and perceptual ability, fMRIa for inverted faces was used as a covariate to control for individual differences in the magnitude of the low-level feature adaptation effect.

Correlation of face discrimination accuracy and fMRIa

First we calculated the magnitude of fMRIa by subtracting the BOLD response during RepT from that of AltT for each subject and area separately. Since we found a strong positive correlation between the fMRIa of the left and right hemisphere homologues of each tested area $\left(r_{(18)}=0.75, p\right.$ $<0.001, \mathrm{CI}=\left[\begin{array}{l}0.350 .94\end{array}\right]$, number of outliers $(\mathrm{NO})=2 ; \mathrm{r}_{(19)}=0.90, \mathrm{p}<0.001, \mathrm{CI}=\left[\begin{array}{ll}0.80 & 0.96\end{array}\right]$, $\mathrm{NO}=1$ and $\mathrm{r}_{(21)}=0.51, \mathrm{p}=0.012, \mathrm{CI}=\left[\begin{array}{ll}0.30 & 0.72\end{array}\right], \mathrm{NO}=1$ for the FFA, OFA, and EBA, respectively), we averaged fMRIa across hemispheres to test for its correlation with face discrimination accuracy. To control for the individual differences in low-level visual feature processing and overall object perception ability, we regressed out the fMRIa and identity discrimination performance for inverted faces from those for upright faces, respectively, before calculating correlations between these two measures. The Skipped Pearson correlation analysis revealed a strong positive correlation between fMRIa and identity discrimination performance in the FFA (Fig. 3a; $\mathrm{r}_{(16)}=0.72, \mathrm{p}<0.001, \mathrm{CI}=[0.410 .91]$, NO $=0$ ) and OFA (Fig. 3b; $\mathrm{r}_{(18)}=0.59$, $\mathrm{p}=0.006, \mathrm{CI}=[0.320 .83], \mathrm{NO}=0$ ), but not in the EBA (Fig. $3 \mathrm{c} ; \mathrm{r}_{(18)}=0.17, \mathrm{p}=0.470, \mathrm{CI}=$ $[-0.230 .58], \mathrm{NO}=1)$. The magnitude of correlation for the FFA and OFA with behavior was not different $\left(C I=\left[\begin{array}{lll}-0.30 & 0.35\end{array}\right]\right)$ and significantly greater than that for the EBA $\left(C I=\left[\begin{array}{ll}0.10 & 1.08\end{array}\right]\right.$ and $\mathrm{CI}=\left[\begin{array}{ll}0.09 & 1.02\end{array}\right]$ for the FFA and OFA, respectively). These findings suggest that genuine faceselective perceptual ability is associated with the fMRIa only in the core face processing areas.

Correlation of fMRIa among the FFA, OFA, and EBA 
To test whether fMRIa reflects common or different underlying mechanisms in the tested visual cortical areas, we calculated Skipped Pearson pairwise correlations of fMRIa magnitudes among the three regions after regressing out fMRIa for inverted faces. The results revealed that the magnitude of fMRIa in the FFA correlates positively and strongly with that of the OFA (Fig. 4a; $\left.\mathrm{r}_{(15)}=0.81, \mathrm{p}<0.001, \mathrm{CI}=[0.590 .94], \mathrm{NO}=0\right)$, but not with that of the EBA (Fig. $4 \mathrm{~b} ; \mathrm{r}_{(15)}=$ $0.05, \mathrm{p}=0.841, \mathrm{CI}=[-0.470 .64], \mathrm{NO}=2)$. Furthermore, the strength of the correlation between FFA and OFA is significantly larger than between the FFA and EBA (CI = [0.18 0.97]). The fMRIa in the OFA showed a moderate, but significant correlation with that in the EBA (Fig. 4c; $\left.\mathrm{r}_{(16)}=0.53, \mathrm{p}=0.025, \mathrm{CI}=\left[\begin{array}{ll}0.10 & 0.83\end{array}\right], \mathrm{NO}=2\right)$, and the magnitude of this correlation did not differ significantly from that of the OFA and FFA $\left(\mathrm{CI}=\left[\begin{array}{ll}0.15 & 0.59\end{array}\right]\right)$. These findings imply that fMRIa might involve different components: one is mediated by neural mechanisms that are specific to the core face processing network and another which affects the fMRI responses in the OFA and EBA, but not in FFA.

\section{Discussion}

The major results of the current study can be summarized as follows: (1) The magnitude of fMRIa measured in the FFA and OFA, but not in the EBA, correlates positively with the behavioral performance of participants in a demanding face discrimination task. The higher the magnitude of the fMRIa for repeated faces, the better the face identity discrimination performance. (2) The observed fMRIa correlates between OFA and FFA, as well as between OFA and EBA, but not between FFA and EBA. These findings suggest that there is a faceselective repetition-induced fMRIa within the core face processing network composed of the FFA and OFA, which reflects adaptive face processing mechanisms that are closely associated to face identity perception.

Network specific fMRIa

The fact that the observed fMRIa correlated between OFA and FFA, also between OFA and EBA, but not between FFA and EBA allows for multiple conclusions. First, it supports further the close connection between OFA and FFA (Rossion 2008). Recent studies suggest that the OFA and FFA are closely and reciprocally connected to each other (Davies-Thompson and Andrews 2012; Gschwind et al. 2012; Nagy et al. 2012; Zhen et al. 2013) and the current results provide 
further functional evidence of this connection by showing that even the response reduction, signaling the sensitivity of neurons to repetitions, is related in the two areas. Our results are in agreement with previous findings from patients with acquired prosopagnosia showing that despite the preserved preferential activation for faces, adaptation effects for face identity in the right FFA are absent following lesions encompassing the right OFA (Schiltz et al. 2006; Steeves et al. 2009). This implies that fMRIa in the right FFA is the result of an intact re-entrant processing loop between these two regions. Furthermore, the close correlation of fMRIa in FFA and OFA supports the conclusion of Ewbank et al. (2013). These authors found that the repetition of a face having the same or different size affected the forward (OFA-to-FFA) and backward (FFA-toOFA) connections specifically. Authors suggested that the fMRIa of a given region reflects the change of reciprocal (forward and backward) cross-region connectivity rather than merely the neural changes within that region. Second, the different correlation patterns we found between fMRIa in the OFA, FFA, and EBA imply that fMRIa might involve different components: one is mediated by neural mechanisms specific for the core face processing network composed of the FFA and OFA and another which affects fMRI responses in the OFA and EBA, but not in FFA. This, in turn, also confirms previous results that suggest that the EBA is not part of the core face processing cortical network (Haxby et al. 2000).

The fact that we found a significant fMRIa in the EBA as well might be surprising for the first glance. Indeed, if an area shows adaptation after being exposed to its less- or non-preferred stimulus (i.e. a face for the body-part sensitive neurons) is surprising if one only considers response fatigue as the neural mechanisms of RS. Firing rate fatigue indeed predicts that the magnitude of adaptation essentially depends on the firing rate of that given neuron to the adapter stimulus. In other words, the larger the response for the adapter, the larger RS one should observe. However, recent single-cell studies disagree with this logic. Liu et al. (2009) reported that the magnitude of RS does not correlate with the trial-to-trial firing rate of a neuron. Moreover, Baene and Vogels (2010) showed that the degree of RS can even be inversely related to the response magnitude, given for the adapter stimulus. Finally, Sawamura et al. (2006) showed that RS can be different for two different adapter stimuli that otherwise elicit the same response magnitude in the neuron. Altogether, these results question the direct relationship between stimulus preference and the magnitude of elicited RS. Therefore, it is possible that face stimuli, which elicit a significant response in the EBA as well (Pinsk et al. 2009; Spiridon et al. 
2006), elicit fMRIa as well.

fMRIa is associated with discrimination performance

The relationship of occipito-temporal activity with behavioral performance in visual stimulus processing was extensively addressed in the past. The activity of FFA and/or OFA has been related to the detection, recognition, or discrimination of faces (Grill-Spector et al. 2000; GrillSpector et al. 2004; Hermann et al. 2015; Nestor et al. 2008). Huang et al. (2014) measured face recognition in an old/new paradigm and found that the participants' recognition ability correlated with the face selectivity of the FFA and OFA, measured by estimating the differential response of the areas for face and non-face object stimuli. However, a very recent study which directly addressed the relationship between face identity memory and face selectivity in the FFA, failed to find correlation between these two measures, except when correlating performance for the most difficult trials including noisy images with the activity in the center region of the FFA (McGugin and Gauthier 2015). This suggests that both the nature of the behavioral task (e.g. perceptual or memory) and task difficulty might affect whether an association between behavioral and neural measures will be observed. Furthermore, the way FFA face sensitivity is measured appears to be similarly important. The findings of Nasr and Tootell (2012) support this conclusion. Authors measured the activity of FFA and of the anterior face patch at the anterior tip of the collateral sulcus and found that only the activity of the more anterior area correlated with response accuracy. Previous results indicate that measuring face sensitivity by fMRI adaptation might be more suitable to uncover relationship between FFA activity and face perception (Goh et al. 2010; Jiang et al. 2013).

So far only one study tried to correlate the repetition related signal reduction to behavioral performance. Furl et al. (2011) tested developmental prosopagnosics and healthy controls and correlated their face identification ability with identity and facial expression specific fMRIa. Authors found neither clear group differences nor any correlation for fMRIa with face identification. Also, Avidan et al. (2005) found normal fMRIa in the FFA and OFA in developmental prosopagnosics and suggested that the fMRIa in these regions is not sufficient for normal face perception ability. These results might seem to contradict those of the current study. However, the approach of the current and the previous studies is sufficiently different to explain the opposing results. While in the current study participants performed a demanding perceptual 
face discrimination task, Furl et al. (2011) used an extensive test battery and PCA analysis to compute a factor score and used this score as a covariate in the regression analysis of the fMRI data. As the test battery contained several perceptual and memory-related tests, it is likely that their behavioral measure reflects more complex face encoding processes as compared to the task applied in the current study. Therefore, it is possible that the fMRIa of the occipito-temporal areas is associated more closely with perceptual than with higher-level, associative or memoryrelated functions. This, in turn, would explain the apparently discrepant results of the Furl et al. (2011) and the current study. Avidan et al. (2005), on the other hand, used blocks of 12 different or identical faces to elicit fMRIa. Therefore, it is possible that the resulting neural adaptation is less sensitive to inter-individual differences than the fMRIa elicited by short presentations of pairs of stimuli in the current study.

We observed significant correlation of behavioral performance with fMRIa in both OFA and FFA. Traditional models of face perception (Haxby et al. 2000; Pitcher et al. 2011) assume a hierarchical model where information flow from early visual cortices towards the OFA is responsible for face detection and categorization, and the FFA and the superior temporal sulcus (STS) represents a higher-level face encoding, where identification and processing of facial expressions occurs. However, the simple feed-forward processing of faces is questioned by recent prosopagnosic (Schiltz et al. 2006; Steeves et al. 2009) and transcranial magnetic stimulation (Solomon-Harris et al. 2013) studies. Altogether, these recent results support a more parallel, non-hierarchical model where OFA and FFA are strongly connected (for a review see Duchaine and Yovel 2015). The similar correlation of FFA and OFA with behavioral performance supports this conclusion.

The neural basis of RS is unclear as of today and this complicates its association to behavioral measures. It is nonetheless generally assumed that RS reflects the specific encoding of the repeated feature or stimulus. In the current study, we measured the fMRI correlate of neural RS for the repetition of the same images with a strong variation in size to reduce low-level image feature adaptation. Furthermore, we controlled for the individual differences in low-level visual feature processing and overall object perception ability by using a regression-based approach which provides a more precise and fine-grained picture of the relationship between fMRI adaptation within the core face processing network and genuine face-selective perceptual ability. 
Whether this relationship is merely correlational or causal will require further studies, possibly combining neuroimaging and brain-stimulation techniques. The causal nature of this relationship, however, is suggested by a recent study. Yang et al. (2016) tested the same acquired prosopagnosic patient as Schiltz et al. (2006) and Steeves et al. (2009). Their findings suggested that the right anterior temporal lobe contains image-invariant face representations (signaled by normal RS) that can persist despite the absence of RS in the right FFA and OFA, but this representation is not sufficient for normal face recognition.

It should be noted that for the current study we only used the data from the blocks with high repetition probabilities of Grotheer et al. (2014) as fMRIa was only measurable within these blocks. One can argue that the observed RS is more related to the implicit capacity of the participants to detect the probability of repetitions than to face perception per se. The fact that in spite of this confound we did find a strong and significant relationship between behavioral performance and RS suggests that this confound is unable to interact with the strong correlation of RS and face discrimination performance. It can be reasoned that within the framework of predictive coding models of perception (Friston 2005), a good generative model of faces can produce better predictions of subsequent stimulations, which leads to better performance and reduced concomitant prediction error unit activity, i.e. fMRIa. If one accepts this argument then the likelihood of finding a relationship between behavior and fMRIa is more likely in the repetition blocks, where the expectation of repetition reduces uncertainty and enhances predictions and therefore the magnitude of fMRIa (Summerfield et al. 2008), compared to blocks where such repetitions are surprising. Nonetheless, this should be taken into account in future studies which should elicit RS in blocks with different statistics as well.

In conclusion, the current study has explored the behavioral relevance of the well-known phenomenon of repetition suppression (RS) for face images. We found that the RS as measured with BOLD fMRI in the core face processing areas, namely in the fusiform face area (FFA) and occipital face area (OFA) is closely associated and predicts individual differences in face perception ability suggesting functionally relevant repetition suppression processes involved in face perception. 


\section{Acknowledgements}

We thank Balázs Knakker, Viktor Gál, and Béla Weiss for their help and advice during data analysis and also for their comments on the manuscript. We also would like to thank the anonymous reviewers for their valuable comments and suggestions.

\section{Compliance with ethical standards}

\section{Funding}

This work was supported by Deutsche Forschungsgemeinschaft Grant (KO 3918/1-2; 2-2). P.H. and Z.V. were supported by a grant from the Hungarian Brain Research Program (KTIA_13_NAP-A-I/18).

\section{Conflict of interest}

Petra Hermann, Mareike Grotheer, Gyula Kovács, and Zoltán Vidnyánszky declare that they have no conflict of interest.

\section{Ethical approval}

All procedures performed in studies involving human participants were in accordance with the ethical standards of the institutional and/or national research committee and with the 1964 Helsinki declaration and its later amendments or comparable ethical standards.

Informed consent

Informed consent was obtained from all patients for being included in the study. Written informed consent was obtained from persons depicted in the photographs.

\section{References}

Andrews, T. J., \& Ewbank, M. P. (2004). Distinct representations for facial identity and changeable aspects of faces in the human temporal lobe. NeuroImage, 23(3), 905-913. doi:10.1016/j.neuroimage.2004.07.060

Auksztulewicz, R., \& Friston, K. (2016). Repetition suppression and its contextual determinants 
in predictive coding. Cortex, 80, 125-140. doi:10.1016/j.cortex.2015.11.024

Avidan, G., Hasson, U., Malach, R., \& Behrmann, M. (2005). Detailed Exploration of Facerelated Processing in Congenital Prosopagnosia: 2. Functional Neuroimaging Findings. Journal of Cognitive Neuroscience, 17(7), 1150-1167. doi:10.1162/0898929054475145

Baene, W. D., \& Vogels, R. (2010). Effects of Adaptation on the Stimulus Selectivity of Macaque Inferior Temporal Spiking Activity and Local Field Potentials. Cerebral Cortex, 20(9), 2145-2165. doi:10.1093/cercor/bhp277

Baylis, G. C., \& Rolls, E. T. (1987). Responses of neurons in the inferior temporal cortex in short term and serial recognition memory tasks. Experimental Brain Research, 65(3), 614-622. doi:10.1007/BF00235984

Brainard, D. H. (1997). The Psychophysics Toolbox. Spatial Vision, 10(4), 433-436. doi:10.1163/156856897X00357

Bunzeck, N., \& Thiel, C. (2016). Neurochemical modulation of repetition suppression and novelty signals in the human brain. Cortex, 80, 161-173. doi:10.1016/j.cortex.2015.10.013

Dakin, S. C., Hess, R. F., Ledgeway, T., \& Achtman, R. L. (2002). What causes non-monotonic tuning of fMRI response to noisy images? Current Biology, 12(14), R476-R477. doi:10.1016/S0960-9822(02)00960-0

Davies-Thompson, J., \& Andrews, T. J. (2012). Intra- and interhemispheric connectivity between face-selective regions in the human brain. Journal of Neurophysiology, 108(11), 30873095. doi:10.1152/jn.01171.2011

Downing, P. E., Jiang, Y., Shuman, M., \& Kanwisher, N. (2001). A Cortical Area Selective for Visual Processing of the Human Body. Science, 293(5539), 2470-2473. doi:10.1126/science.1063414

Duchaine, B., \& Yovel, G. (2015). A Revised Neural Framework for Face Processing. Annual Review of Vision Science, 1(1), 393-416. doi:10.1146/annurev-vision-082114-035518

Ewbank, M. P., Henson, R. N., Rowe, J. B., Stoyanova, R. S., \& Calder, A. J. (2013). Different Neural Mechanisms within Occipitotemporal Cortex Underlie Repetition Suppression across Same and Different-Size Faces. Cerebral Cortex, 23(5), 1073-1084. doi:10.1093/cercor/bhs070

Friston, K. (2005). A theory of cortical responses. Philosophical Transactions of the Royal 
Society of London B: Biological Sciences, 360(1456), 815-836. doi:10.1098/rstb.2005.1622

Furl, N., Garrido, L., Dolan, R., Driver, J., \& Duchaine, B. (2011). Fusiform gyrus faceselectivity reflects facial recognition ability. Journal of cognitive neuroscience, 23(7), 1723-1740. doi:10.1162/jocn.2010.21545

Ganel, T., Gonzalez, C. L. R., Valyear, K. F., Culham, J. C., Goodale, M. A., \& Köhler, S. (2006). The relationship between fMRI adaptation and repetition priming. NeuroImage, 32(3), 1432-1440. doi:10.1016/j.neuroimage.2006.05.039

Gilaie-Dotan, S., Gelbard-Sagiv, H., \& Malach, R. (2010). Perceptual shape sensitivity to upright and inverted faces is reflected in neuronal adaptation. NeuroImage, 50(2), 383-395. doi:10.1016/j.neuroimage.2009.12.077

Goh, J. O., Suzuki, A., \& Park, D. C. (2010). Reduced neural selectivity increases fMRI adaptation with age during face discrimination. NeuroImage, 51(1), 336-344. doi:10.1016/j.neuroimage.2010.01.107

Grill-Spector, K., Henson, R., \& Martin, A. (2006). Repetition and the brain: neural models of stimulus-specific effects. Trends in Cognitive Sciences, 10(1), 14-23. doi:10.1016/j.tics.2005.11.006

Grill-Spector, K., Knouf, N., \& Kanwisher, N. (2004). The fusiform face area subserves face perception, not generic within-category identification. Nature Neuroscience, 7(5), 555562. doi:10.1038/nn1224

Grill-Spector, K., Kushnir, T., Hendler, T., \& Malach, R. (2000). The dynamics of objectselective activation correlate with recognition performance in humans. Nature Neuroscience, 3(8), 837-843. doi:10.1038/77754

Grotheer, M., Hermann, P., Vidnyánszky, Z., \& Kovács, G. (2014). Repetition probability effects for inverted faces. NeuroImage, 102, Part 2, 416-423. doi:10.1016/j.neuroimage.2014.08.006

Grotheer, M., \& Kovács, G. (2016). Can predictive coding explain repetition suppression? Cortex, 80, 113-124. doi:10.1016/j.cortex.2015.11.027

Gschwind, M., Pourtois, G., Schwartz, S., Ville, D. V. D., \& Vuilleumier, P. (2012). WhiteMatter Connectivity between Face-Responsive Regions in the Human Brain. Cerebral Cortex, 22(7), 1564-1576. doi:10.1093/cercor/bhr226 
Haxby, J. V., Hoffman, E. A., \& Gobbini, M. I. (2000). The distributed human neural system for face perception. Trends in Cognitive Sciences, 4(6), 223-233. doi:10.1016/S13646613(00)01482-0

Hecaen, H., \& Angelergues, R. (1962). Agnosia for faces (prosopagnosia). Archives of Neurology, 7, 92-100.

Henson, R. N. A. (2003). Neuroimaging studies of priming. Progress in Neurobiology, 70(1), $53-$ 81.

Henson, R. N. A., \& Rugg, M. D. (2003). Neural response suppression, haemodynamic repetition effects, and behavioural priming. Neuropsychologia, 41(3), 263-270.

Hermann, P., Bankó, É. M., Gál, V., \& Vidnyánszky, Z. (2015). Neural Basis of Identity Information Extraction from Noisy Face Images. The Journal of Neuroscience, 35(18), 7165-7173. doi:10.1523/JNEUROSCI.3572-14.2015

Horner, A. J., \& Henson, R. N. (2011). Repetition suppression in occipitotemporal cortex despite negligible visual similarity: Evidence for postperceptual processing? Human Brain Mapping, 32(10), 1519-1534. doi:10.1002/hbm.21124

Huang, L., Song, Y., Li, J., Zhen, Z., Yang, Z., \& Liu, J. (2014). Individual differences in cortical face selectivity predict behavioral performance in face recognition. Frontiers in Human Neuroscience, 8, 483. doi:10.3389/fnhum.2014.00483

Jiang, X., Bollich, A., Cox, P., Hyder, E., James, J., Gowani, S. A., et al. (2013). A quantitative link between face discrimination deficits and neuronal selectivity for faces in autism. NeuroImage: Clinical, 2, 320-331. doi:10.1016/j.nic1.2013.02.002

Jonas, J., Rossion, B., Krieg, J., Koessler, L., Colnat-Coulbois, S., Vespignani, H., et al. (2014). Intracerebral electrical stimulation of a face-selective area in the right inferior occipital cortex impairs individual face discrimination. NeuroImage, 99, 487-497. doi:10.1016/j.neuroimage.2014.06.017

Kaiser, D., Walther, C., Schweinberger, S. R., \& Kovács, G. (2013). Dissociating the neural bases of repetition-priming and adaptation in the human brain for faces. Journal of Neurophysiology, 110(12), 2727-2738. doi:10.1152/jn.00277.2013

Kaliukhovich, D. A., \& Vogels, R. (2011). Stimulus Repetition Probability Does Not Affect Repetition Suppression in Macaque Inferior Temporal Cortex. Cerebral Cortex, 21(7), 1547-1558. doi:10.1093/cercor/bhq207 
Kaliukhovich, D. A., \& Vogels, R. (2012). Stimulus repetition affects both strength and synchrony of macaque inferior temporal cortical activity. Journal of Neurophysiology, 107(12), 3509-3527. doi:10.1152/jn.00059.2012

Kanwisher, N., McDermott, J., \& Chun, M. M. (1997). The Fusiform Face Area: A Module in Human Extrastriate Cortex Specialized for Face Perception. The Journal of Neuroscience, 17(11), 4302-4311.

Kovács, G., Iffland, L., Vidnyánszky, Z., \& Greenlee, M. W. (2012). Stimulus repetition probability effects on repetition suppression are position invariant for faces. NeuroImage, 60(4), 2128-2135. doi:10.1016/j.neuroimage.2012.02.038

Kovács, G., Kaiser, D., Kaliukhovich, D. A., Vidnyánszky, Z., \& Vogels, R. (2013). Repetition Probability Does Not Affect fMRI Repetition Suppression for Objects. The Journal of Neuroscience, 33(23), 9805-9812. doi:10.1523/JNEUROSCI.3423-12.2013

Kovács, G., \& Vogels, R. (2014). When does repetition suppression depend on repetition probability? Frontiers in Human Neuroscience, 8, 685. doi:10.3389/fnhum.2014.00685

Larsson, J., \& Smith, A. T. (2012). fMRI Repetition Suppression: Neuronal Adaptation or Stimulus Expectation? Cerebral Cortex, 22(3), 567-576. doi:10.1093/cercor/bhr119

Liu, Y., Murray, S. O., \& Jagadeesh, B. (2009). Time Course and Stimulus Dependence of Repetition-Induced Response Suppression in Inferotemporal Cortex. Journal of Neurophysiology, 101(1), 418-436. doi:10.1152/jn.90960.2008

Magnussen, S. (2000). Low-level memory processes in vision. Trends in Neurosciences, 23(6), 247-251. doi:10.1016/S0166-2236(00)01569-1

McGugin, R. W., \& Gauthier, I. (2015). The reliability of individual differences in face-selective responses in the fusiform gyrus and their relation to face recognition ability. Brain Imaging and Behavior, 1-12. doi:10.1007/s11682-015-9467-4

Meadows, J. C. (1974). The anatomical basis of prosopagnosia. Journal of Neurology, Neurosurgery, and Psychiatry, 37(5), 489-501.

Miller, E. K., Gochin, P. M., \& Gross, C. G. (1991). Habituation-like decrease in the responses of neurons in inferior temporal cortex of the macaque. Visual Neuroscience, 7(4), 357-362.

Miller, E. K., Li, L., \& Desimone, R. (1991). A neural mechanism for working and recognition memory in inferior temporal cortex. Science (New York, N.Y.), 254(5036), 1377-1379.

Nagy, K., Greenlee, M. W., \& Kovács, G. (2012). The lateral occipital cortex in the face 
perception network: an effective connectivity study. Frontiers in Psychology, 3, 141. doi:10.3389/fpsyg.2012.00141

Nasr, S., \& Tootell, R. B. (2012). Role of Fusiform and Anterior Temporal Cortical Areas in Facial Recognition. NeuroImage, 63(3), 1743-1753. doi:10.1016/j.neuroimage.2012.08.031

Nestor, A., Vettel, J. M., \& Tarr, M. J. (2008). Task-Specific Codes for Face Recognition: How they Shape the Neural Representation of Features for Detection and Individuation. PLoS ONE, 3(12), e3978. doi:10.1371/journal.pone.0003978

Pelli, D. G. (1997). The VideoToolbox software for visual psychophysics: transforming numbers into movies. Spatial Vision, 10(4), 437-442. doi:10.1163/156856897X00366

Pernet, C. R., Wilcox, R., \& Rousselet, G. A. (2012). Robust correlation analyses: false positive and power validation using a new open source matlab toolbox. Frontiers in Psychology, 3, 606. doi:10.3389/fpsyg.2012.00606

Pinsk, M. A., Arcaro, M., Weiner, K. S., Kalkus, J. F., Inati, S. J., Gross, C. G., \& Kastner, S. (2009). Neural Representations of Faces and Body Parts in Macaque and Human Cortex: A Comparative fMRI Study. Journal of Neurophysiology, 101(5), 2581-2600. doi:10.1152/jn.91198.2008

Pitcher, D., Walsh, V., \& Duchaine, B. (2011). The role of the occipital face area in the cortical face perception network. Experimental Brain Research, 209(4), 481-493. doi:10.1007/s00221-011-2579-1

Pitcher, D., Walsh, V., Yovel, G., \& Duchaine, B. (2007). TMS Evidence for the Involvement of the Right Occipital Face Area in Early Face Processing. Current Biology, 17(18), 15681573. doi:10.1016/j.cub.2007.07.063

Ringo, J. L. (1996). Stimulus specific adaptation in inferior temporal and medial temporal cortex of the monkey. Behavioural Brain Research, 76(1-2), 191-197. doi:10.1016/01664328(95)00197-2

Rossion, B. (2008). Constraining the cortical face network by neuroimaging studies of acquired prosopagnosia. NeuroImage, 40(2), 423-426. doi:10.1016/j.neuroimage.2007.10.047

Rossion, B., Hanseeuw, B., \& Dricot, L. (2012). Defining face perception areas in the human brain: A large-scale factorial fMRI face localizer analysis. Brain and Cognition, 79(2), 138-157. doi:10.1016/j.bandc.2012.01.001 
Sawamura, H., Orban, G. A., \& Vogels, R. (2006). Selectivity of Neuronal Adaptation Does Not Match Response Selectivity: A Single-Cell Study of the fMRI Adaptation Paradigm. Neuron, 49(2), 307-318. doi:10.1016/j.neuron.2005.11.028

Sayres, R., \& Grill-Spector, K. (2006). Object-Selective Cortex Exhibits PerformanceIndependent Repetition Suppression. Journal of Neurophysiology, 95(2), 995-1007. doi:10.1152/jn.00500.2005

Schiltz, C., Sorger, B., Caldara, R., Ahmed, F., Mayer, E., Goebel, R., \& Rossion, B. (2006). Impaired Face Discrimination in Acquired Prosopagnosia Is Associated with Abnormal Response to Individual Faces in the Right Middle Fusiform Gyrus. Cerebral Cortex, 16(4), 574-586. doi:10.1093/cercor/bhj005

Sobotka, S., \& Ringo, J. L. (1994). Stimulus specific adaptation in excited but not in inhibited cells in inferotemporal cortex of macaque. Brain Research, 646(1), 95-99.

Soldan, A., Habeck, C., Gazes, Y., \& Stern, Y. (2010). Neural mechanisms of repetition priming of familiar and globally unfamiliar visual objects. Brain Research, 1343, 122-134. doi:10.1016/j.brainres.2010.04.071

Solomon-Harris, L. M., Mullin, C. R., \& Steeves, J. K. E. (2013). TMS to the "occipital face area" affects recognition but not categorization of faces. Brain and Cognition, 83(3), 245251. doi:10.1016/j.bandc.2013.08.007

Spiridon, M., Fischl, B., \& Kanwisher, N. (2006). Location and Spatial Profile of CategorySpecific Regions in Human Extrastriate Cortex. Human Brain Mapping, 27(1), 77-89. doi:10.1002/hbm.20169

Steeves, J., Dricot, L., Goltz, H. C., Sorger, B., Peters, J., Milner, A. D., et al. (2009). Abnormal face identity coding in the middle fusiform gyrus of two brain-damaged prosopagnosic $\begin{array}{llll}\text { patients. } & \text { Neuropsychologia, } & \text { 2584-2592. }\end{array}$ doi:10.1016/j.neuropsychologia.2009.05.005

Summerfield, C., Trittschuh, E. H., Monti, J. M., Mesulam, M.-M., \& Egner, T. (2008). Neural repetition suppression reflects fulfilled perceptual expectations. Nature Neuroscience, 11(9), 1004-1006. doi:10.1038/nn.2163

Tiddeman, B., Burt, M., \& Perrett, D. (2001). Prototyping and transforming facial textures for perception research. IEEE Computer Graphics and Applications, 21(5), 42-50. doi:10.1109/38.946630 
Trujillo-Ortiz, A., Hernandez-Walls, R., Barba-Rojo, K., \& Cupul-Magana, L. (2007). HZmvntest: Henze-Zirkler's Multivariate Normality Test. http://www.mathworks.com/matlabcentral/fileexchange/loadFile.do?objectId=17931

Vogels, R. (2016). Sources of adaptation of inferior temporal cortical responses. Cortex, 80, 185195. doi:10.1016/j.cortex.2015.08.024

Ward, E. J., Chun, M. M., \& Kuhl, B. A. (2013). Repetition Suppression and Multi-Voxel Pattern Similarity Differentially Track Implicit and Explicit Visual Memory. The Journal of Neuroscience, 33(37), 14749-14757. doi:10.1523/JNEUROSCI.4889-12.2013

Winston, J. S., Henson, R. N. A., Fine-Goulden, M. R., \& Dolan, R. J. (2004). fMRI-Adaptation Reveals Dissociable Neural Representations of Identity and Expression in Face Perception. Journal of Neurophysiology, 92(3), 1830-1839. doi:10.1152/jn.00155.2004

Yang, H., Susilo, T., \& Duchaine, B. (2016). The Anterior Temporal Face Area Contains Invariant Representations of Face Identity That Can Persist Despite the Loss of Right FFA and OFA. Cerebral Cortex, 26(3), 1096-1107. doi:10.1093/cercor/bhu289

Yin, R. K. (1969). Looking at upside-down faces. Journal of Experimental Psychology, 81(1), 141-145. doi:10.1037/h0027474

Yovel, G., \& Kanwisher, N. (2005). The Neural Basis of the Behavioral Face-Inversion Effect. Current Biology, 15(24), 2256-2262. doi:10.1016/j.cub.2005.10.072

Yovel, G., Tambini, A., \& Brandman, T. (2008). The asymmetry of the fusiform face area is a stable individual characteristic that underlies the left-visual-field superiority for faces. Neuropsychologia, 46(13), 3061-3068. doi:10.1016/j.neuropsychologia.2008.06.017

Zhen, Z., Fang, H., \& Liu, J. (2013). The Hierarchical Brain Network for Face Recognition. PLoS ONE, 8(3), e59886. doi:10.1371/journal.pone.0059886

Zou, G. Y. (2007). Toward using confidence intervals to compare correlations. Psychological Methods, 12(4), 399-413. doi:10.1037/1082-989X.12.4.399 


\section{Figure Captions}

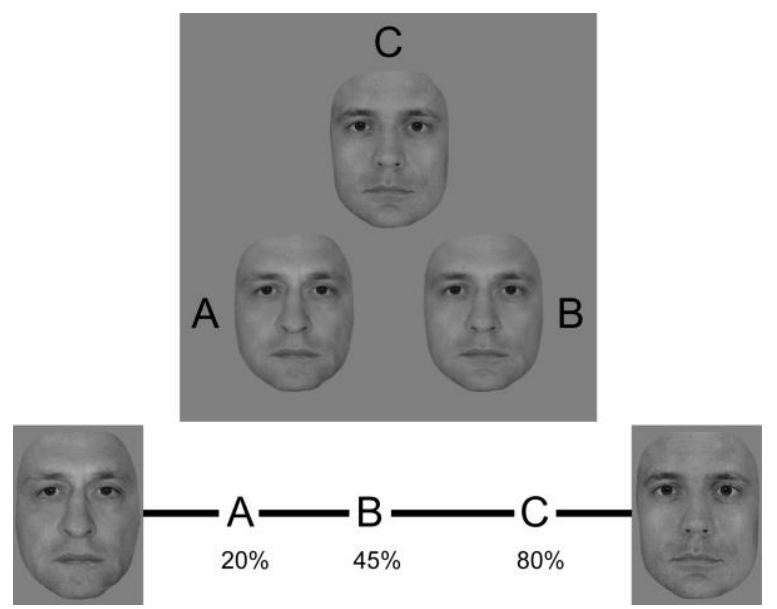

Fig. 1 Example stimulus of the behavioral task performed outside the MRI scanner. By morphing two paired individuals (illustrated on the bottom), we created face triplets (top) including faces at 20/80\% (A), 45/55\% (B) and 80/20\% (C) points of the morph-space and presented in a regular triangular arrangement upright and upside down (inverted). Please note that the letters are only for illustration purposes and were not presented in the actual experiments. 

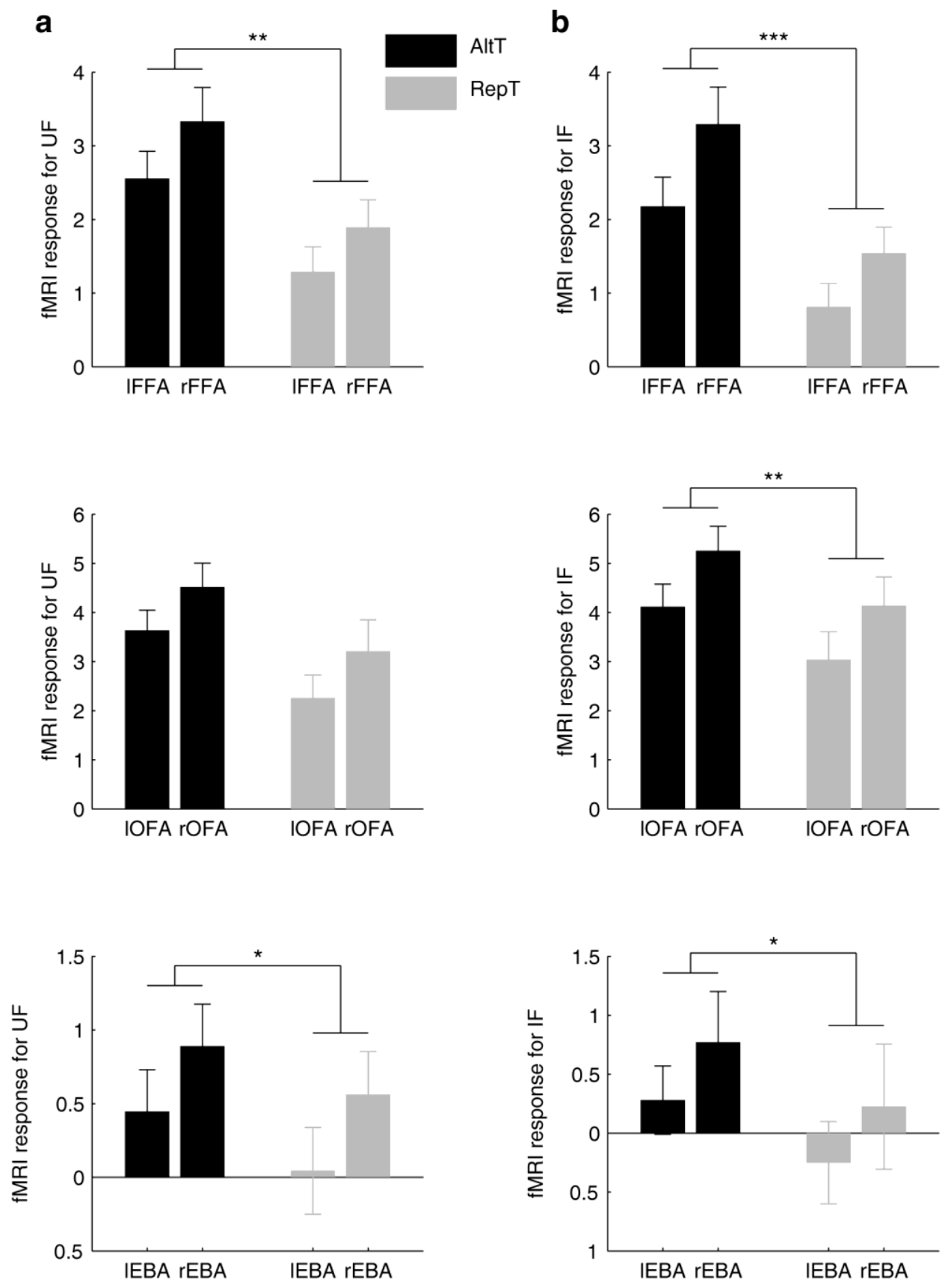

Fig. 2 Average activation ( \pm SE) profiles for the left and right FFA (top), OFA (middle), and EBA (bottom) when faces were presented upright (a) and inverted (b). Black bars represent AltT, gray bars represent RepT. (UF: upright faces; IF: inverted faces; *p $<0.05, * * p<0.01$, ***p $<$ $0.001)$ 
a

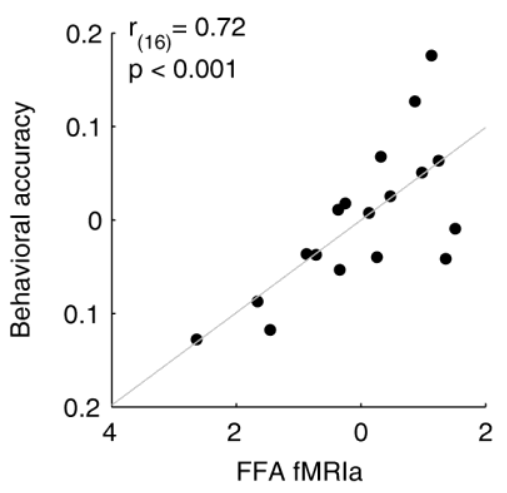

b

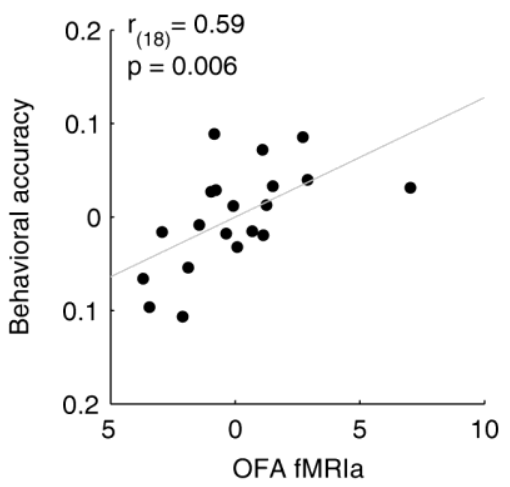

C

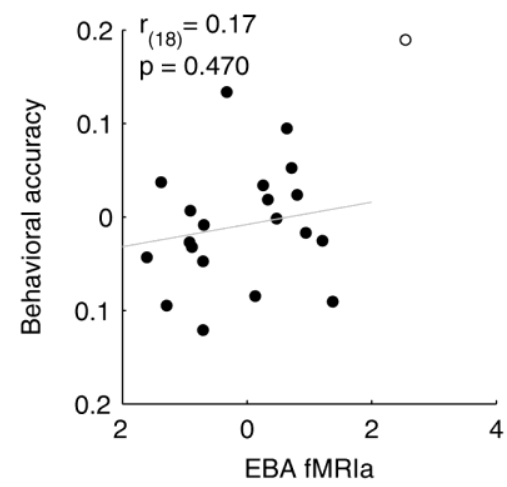

Fig. 3 Correlation between behavioral accuracy and fMRIa for the FFA (a), OFA (b), and EBA (c). Significant correlation was found in the case of the FFA and OFA, but not for the EBA. Due to the regression-based approach (see Methods for details) correlation scatter plots depict residual values on both axes. $y$-axis values denote behavioral accuracy in the face identity discrimination task indexed by the residual correct response ratio. $x$-axis values denote the fMRIa indexed by the residual beta difference in the AltT vs. RepT contrast. Circles represent individual participants; bivariate outliers are marked with open circles. Diagonal line indicates linear least squares fit

a

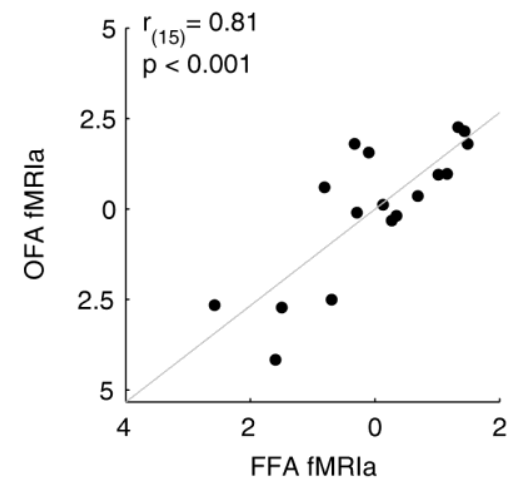

b

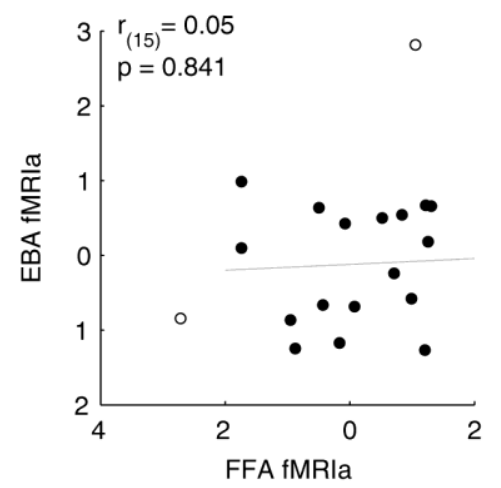

C

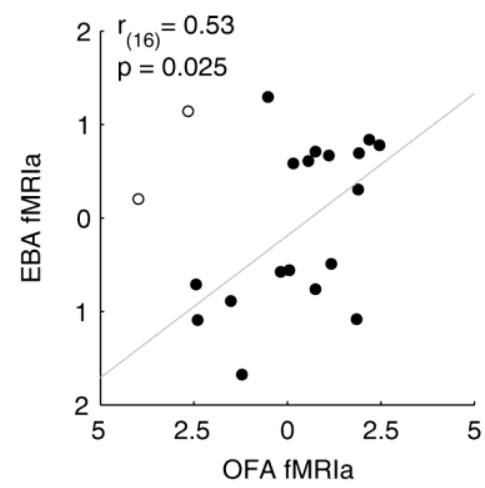

Fig. 4 Correlation between fMRIa observed in the FFA and OFA (a), in the FFA and EBA (b) and in the OFA and EBA (c). Significant correlation was found between the magnitude of fMRIa measured in the FFA and OFA, as well as in the OFA and EBA, but not in the FFA and EBA. Due to the regression-based approach (see Methods for details), correlation scatter plots depict 
residual values on both axes. $y$ - and $x$-axis values denote the fMRIa indexed by the residual beta difference in the AltT vs. RepT contrast. Circles represent individual participants; bivariate outliers are marked with open circles. Diagonal line indicates linear least squares fit 